\title{
An inexpensive open source 3D-printed membrane feeder for human malaria transmission studies
}

\author{
Kathrin Witmer ${ }^{1}$, Ellie Sherrard-Smith², Ursula Straschil ${ }^{1}$, Mark Tunnicliff ${ }^{1}$, Jake Baum ${ }^{1}$ and Michael Delves ${ }^{1 *}$ (i)
}

\begin{abstract}
Background: The study of malaria transmission requires the experimental infection of mosquitoes with Plasmodium gametocytes. In the laboratory, this is achieved using artificial membrane feeding apparatus that simulate body temperature and skin of the host, and so permit mosquito feeding on reconstituted gametocyte-containing blood. Membrane feeders either use electric heating elements or complex glass chambers to warm the infected blood; both of which are expensive to purchase and can only be sourced from a handful of specialized companies. Presented and tested here is a membrane feeder that can be inexpensively printed using 3D-printing technology.

Results: Using the Plasmodium falciparum laboratory strain NF54, three independent standard membrane feeding assays (SMFAs) were performed comparing the 3D-printed feeder against a commercial glass feeder. Exflagellation rates did not differ between the two feeders. Furthermore, no statistically significant difference was found in the oocyst load nor oocyst intensity of Anopheles stephensi mosquitoes (mean oocyst range 1.3-6.2 per mosquito; infection prevalence range $41-79 \%)$.

Conclusions: Open source provision of the design files of the 3D-printed feeder will facilitate a wider range of laboratories to perform SMFAs in laboratory and field settings, and enable them to freely customize the design to their own requirements.
\end{abstract}

Keywords: Malaria, Transmission, Gametocyte, Mosquito, SMFA

\section{Background}

Transmission of malaria from vertebrate host to mosquito is mediated by the mature sexual stages of the Plasmodium life cycle-male and female gametocytes. Gametocytes sense their uptake into the mosquito midgut by a decrease in temperature and the presence of mosquito-derived xanthurenic acid and rapidly differentiate into male and female gametes [1]. Gametes fuse and fertilization ensues, with the resultant motile ookinetes migrating to and through the midgut epithelium, where they develop into oocysts upon contacting the basal lamina. Artificial feeding of mosquitoes using gametocyte-infected blood in a membrane feeding

\footnotetext{
*Correspondence: michael.delves@imperial.ac.uk

${ }^{1}$ Department of Life Sciences, Imperial College, London SW7 2AZ, UK

Full list of author information is available at the end of the article
}

system is a mainstay of Plasmodium transmission stage research to study cell biology, vaccine and antimalarial drug development [2-4]. At its simplest, membrane feeding requires a gametocyte-containing blood meal, a source of heat to maintain the blood at $37{ }^{\circ} \mathrm{C}$ (to ensure gametocytes are not prematurely activated and to simulate body temperature to promote mosquito feeding) and a membrane around the blood to simulate the skin of the host [5]. Two solutions are commonly employed to perform the Standard Membrane Feeding Assay (SMFA): (1) Hemotek ${ }^{\circledR}[6]$, in which infected blood is placed between electrically heated feeder reservoirs and a surrounding membrane. (2) Water-jacketed glass or plastic feeders in which heated water from a circulating water bath passes through the feeder and warms the infected blood sample surrounded with a membrane [7]. This type of

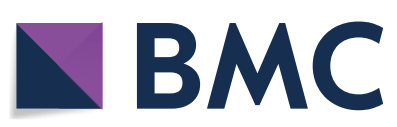

(c) The Author(s) 2018. This article is distributed under the terms of the Creative Commons Attribution 4.0 International License (http://creativecommons.org/licenses/by/4.0/), which permits unrestricted use, distribution, and reproduction in any medium, provided you give appropriate credit to the original author(s) and the source, provide a link to the Creative Commons license, and indicate if changes were made. The Creative Commons Public Domain Dedication waiver (http://creativecommons.org/ publicdomain/zero/1.0/) applies to the data made available in this article, unless otherwise stated. 
glass feeder is most commonly applied in field settings [8] using a standardized protocol [5]. Whilst both are effective, they can be expensive for laboratories with limited resources and can only be obtained from a few suppliers, thus limiting their availability.

3D-printing refers to any process using computer control to create a three-dimensional object. The 3D-printing revolution has opened up professional design and manufacture on a small-scale to mainstream users, enabling rapid transitions from an initial design to the finished product. Presented here is a simple two-piece water-jacketed membrane feeder designed to hold a volume of $500 \mu \mathrm{l}$. The design for the feeder used here is supplied in OBJ format (Additional files 1 and 2), which can be opened in any computer-aided design (CAD) package for 3D-printing-many of which are provided for free download on the Internet. Using the files presented here, the feeder can be 3D-printed directly and inexpensively by stereolithography by any equipped lab or commercial 3D-printing provider. Alternatively, by using a CAD package the size of the feeder can be up- or downscaled to hold more or less volume respectively.

This study validates and compares the acrylic resin 3D-printed feeder to a conventional glass feeder. Exflagellation rates as well as oocyst counts indicate that there is no significant difference between the two, within the statistical power given by triplicate SMFAs used as standard by the research community. The design of the feeder is provided here, enabling others to gain inexpensive access to equipment needed to perform SMFAs and making future modifications or improvements to the design straightforward.

\section{Methods}

\section{Design and production of the membrane feeder}

The design for the membrane feeder was modelled in the free open source CAD modeller Art of Illusion v3.0.2 (http://www.artofillusion.org/) using combined Boolean modelling of simple geometric shapes. The 3D models of the top and bottom halves of the feeder were commercially printed in USP VI medical-grade "Fine Detail Plastic" acrylic resin (VisiJet M3 Crystal) using stereolithography by Shapeways (https://www. shapeways.com/). The 3D modelling files are freely available for download and modification (Additional files 1,2) under a Creative Commons 4.0 Attribution International license. Before first use, both halves of the feeder were washed extensively with tap water to remove any unpolymerized resin and allowed to dry before being glued together with cyanoacrylate cement ("superglue").

\section{Plasmodium falciparum standard membrane feeding assay} (SMFA)

Plasmodium falciparum NF54 gametocytes were prepared by standard methods [7] and between 13-16 days after culture induction were fed to 3-7 days old Anopheles stephensi mosquitoes. All culture manipulations were performed at $37{ }^{\circ} \mathrm{C}$ to prevent premature activation of the gametocytes. Briefly, 200-300 $\mu \mathrm{l}$ of pre-warmed fresh human red blood cells $(\mathrm{RBC})(\mathrm{O}+$ male) were added at the bottom of a $15 \mathrm{ml}$ conical tube, and the gametocyte culture added on top. The mixture was pelleted using a heated centrifuge at $500 \mathrm{rcf}$ for $5 \mathrm{~min}$ at $38^{\circ} \mathrm{C}$. The supernatant was removed with an aspirator and the $\mathrm{RBC} /$ gametocyte pellet was mixed with pre-warmed human serum in a ratio of 2:3. The conventional glass feeder (Dixon Glass) and the 3D-printed feeder were attached to a $38{ }^{\circ} \mathrm{C}$ circulating water bath (Grant Instruments) chained in a loop with silicone tubing (total circulation loop $=\sim 50 \mathrm{~cm}$ ). The bottom of the pre-warmed feeders was covered with Parafilm ${ }^{\circledR}$ stretched in both directions to make a thin membrane. The RBC/gametocyte/serum mixture was equally distributed into both feeders and mosquitoes were allowed to feed for 20-30 min at ambient room temperature of $21^{\circ} \mathrm{C}$.

After mosquito feeding, the gametocyte/RBC/serum mixture was removed into prewarmed microcentrifuge tubes and put on a heating block at $37^{\circ} \mathrm{C}$ to test for exflagellation (see below). The feeder set up was disassembled and feeders were washed with hot water. Exposure to $10 \%$ bleach for 7 consecutive days did not affect the mechanical properties or durability of the 3D-printed feeder and permits a more rigorous decontamination procedure if required for safety reasons such as SMFAs with unscreened blood.

After infection, mosquitoes were maintained at $26{ }^{\circ} \mathrm{C}$ and $80 \%$ humidity. $24 \mathrm{~h}$ post-infection, unfed mosquitoes were removed. After 9 days, mosquito midguts were removed and stained in $0.1 \%$ mercurochrome in PBS for $15 \mathrm{~min}$. Oocysts per midgut were counted at $20 \times$ magnification using a light microscope.

\section{Post-feed exflagellation assay}

After membrane feeding and whilst the feeders were still assembled and warm, the remaining RBC/gametocyte/serum mixture was removed into a microcentrifuge tube and kept at $37{ }^{\circ} \mathrm{C}$ (see above). $10 \mu \mathrm{l}$ of the $\mathrm{RBC} /$ gametocyte/serum mixture was then added to $90 \mu \mathrm{l}$ incomplete ookinete medium $(100 \mu \mathrm{M}$ xanthurenic acid, $2 \mathrm{~g} / \mathrm{l}$ sodium bicarbonate, $50 \mathrm{mg} / \mathrm{l}$ hypoxanthine in RPMI1640-HEPES, pH 7.4) [9], transferred to a FastRead Counting Slide and incubated at room temperature. After 10-15 min, exflagellation centres were counted using 
brightfield microscopy with a $10 \times$ objective three times for each feeder. This number was then multiplied by ten to express exflagellation per $\mathrm{ml}$ of feeder.

\section{Statistical analysis}

Statistical analyses for exflagellation rates and oocyst numbers were performed using GraphPad Prism version 7 or $\mathrm{R}$ version 3.5. Exflagellation was compared using paired t-test; oocyst intensity and prevalence were compared using a zero-inflated Poisson regression to accommodate the distribution of the count data [10]. In addition, a Bayesian approach was used to demonstrate there is no statistical difference between experimental replicates. Zero-inflated Poisson probability distributions were fitted using Hamiltonian Monte Carlo sampling methods [11]. The probability function is:

$$
p\left(y_{n} \mid \theta, \lambda\right)=\left\{\begin{array}{l}
\theta+(1-\theta) \times \text { Poisson }(0 \mid \lambda) \\
(1-\theta) \times \text { Poisson }\left(y_{n} \mid \lambda\right)
\end{array}\right.
$$

where $y$ represents the number of oocysts observed in $n$ mosquitoes, and there is a probability $\theta$ of drawing a zero and a probability $1-\theta$ of drawing from a Poisson distribution with mean parameter $\lambda$. Four chains were initialised to assess the convergence of 2000 iterations, the first 1000 of each were discarded as burn in. The posterior distributions of parameters (4000 iterations) and 95\% Bayesian credible intervals were estimated, posterior checks were performed using shinystan library version 2.5 and visually confirmed to fit the data (Fig. 3). Power calculations of feed data were estimated in $\mathrm{R}$ using the pwr library.

\section{Results}

\section{Assembly and operation of the membrane feeder}

The 3D-printed membrane feeder was designed and manufactured in two parts (Additional files 1,2) - a bottom chamber to accommodate the infected blood sample and circulating heated water, and a top chamber to accommodate connection to the circulating water source (Fig. 1a). To assemble the feeder, the two parts were glued together with cyanoacrylate cement ("superglue") so that the notches in the top part align with the two injection holes in the bottom part (Fig. 1b). To operate, the feeder was connected to a circulating water bath at $38^{\circ} \mathrm{C}$ (to ensure a continual supply of water to warm the feeder) and allowed to equilibrate for $20 \mathrm{~min}$. A piece of Parafilm ${ }^{\circledR}$ stretched thin in both directions was wrapped over the underside of the feeder and a $500 \mu$ l sample containing $\mathrm{RBC} /$ gametocytes/serum was introduced via the injection holes (Fig. 1c).

\section{D-printed membrane feeder supports the transmission of $P$. falciparum to mosquitoes}

The feeder was tested head-to-head against a commercial glass membrane feeder. The $\mathrm{RBC} /$ gametocyte/serum mixture was divided equally between the two. Separate pots of An. stephensi mosquitoes were allowed to feed on both feeders and residual blood was collected to assess male gametocyte exflagellation levels by quantification in a haemocytometer. With three independent biological replicates derived from different gametocyte cultures and different mosquito generations, both the glass and 3D-printed feeders showed no significant differences in exflagellation post-feeding (paired t test; $\mathrm{p}=0.26,0.23$ and 0.88 respectively for replicates $1-3$ ) (Fig. 2a). This suggests both that the heat transfer from the circulating water is sufficient to preserve gametocyte viability and that the acrylic resin photopolymer material of the $3 \mathrm{D}$-printed feeder is non-toxic and does not affect the parasites during feeding (Fig. 2a). Nine days later when An. stephensi oocyst burden was assessed (Fig. 2b), it was found that there was no significant difference in oocyst intensity (Zero-inflated regression (Binomial with logit link): $\mathrm{p}=0.994,0.188$ and 0.756 , respectively for replicates $1-3$ ). The Bayesian analysis showed clearly that in each experimental replicate of either membrane feeder, there is no difference in the data distribution or the parameter estimates that can describe these data. Even with 4000 posterior estimates for the parameters $\theta$ and $\lambda$, no difference was observed in the range of estimates for any of the experimental replicates (Fig. 3). The infection prevalence of An. stephensi mosquitoes was not statistically different between each feeder in all three biological replicates (Fisher's Exact test; $\mathrm{p} \geq 0.99,0.28,>0.99$, respectively for replicates $1-3$ ) (Fig. 2b). Assuming a statistical power of 0.8 , a difference of $>65 \%$ in prevalence between the two feeders in all three replicates would be significant with $95 \%$ confidence.

\section{Discussion}

Under standard SMFA conditions, the 3D-printed, acrylic resin photopolymer membrane feeder was found to give equally successful $P$. falciparum infections in $A n$. stephensi mosquitoes when compared to a glass counterpart but at a third of the cost ( $€ 20$ versus $€ 58$ ).

Like glass feeders, the printed unit is robust and reusable, providing a cost-effective alternative to existing solutions. The only operational difference found in testing of the 3D printed feeder compared to conventional protocols was that it required extended washing in tap water after feeding experiments to remove residual blood due to the rough unfinished surface of the acrylic resin. This could be mitigated in the future by polishing 
a
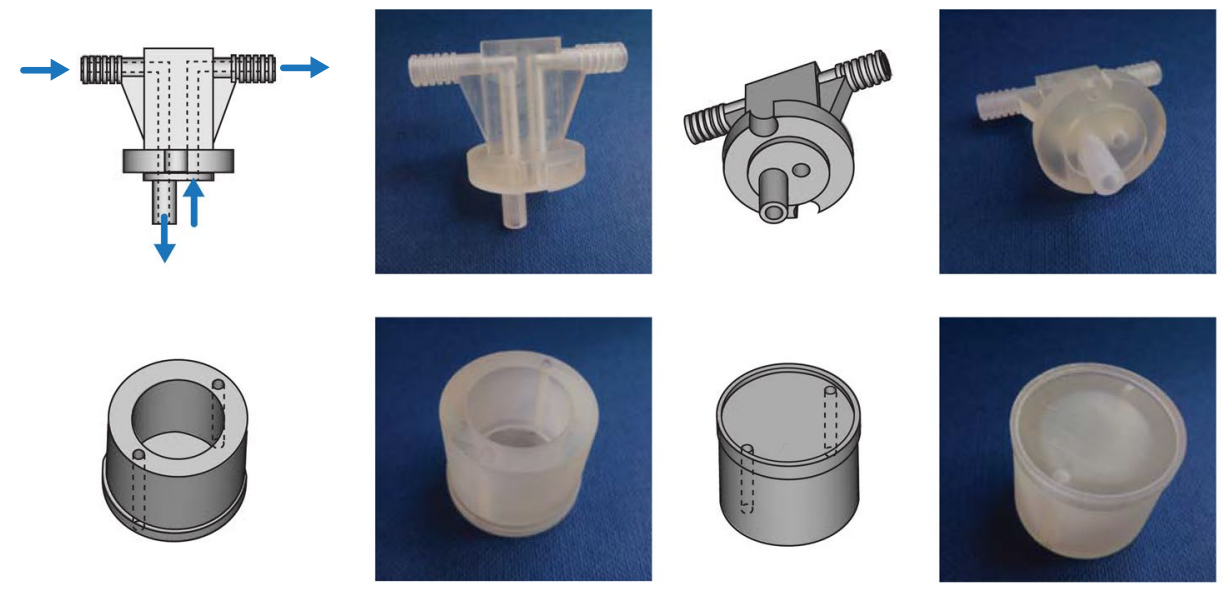

b
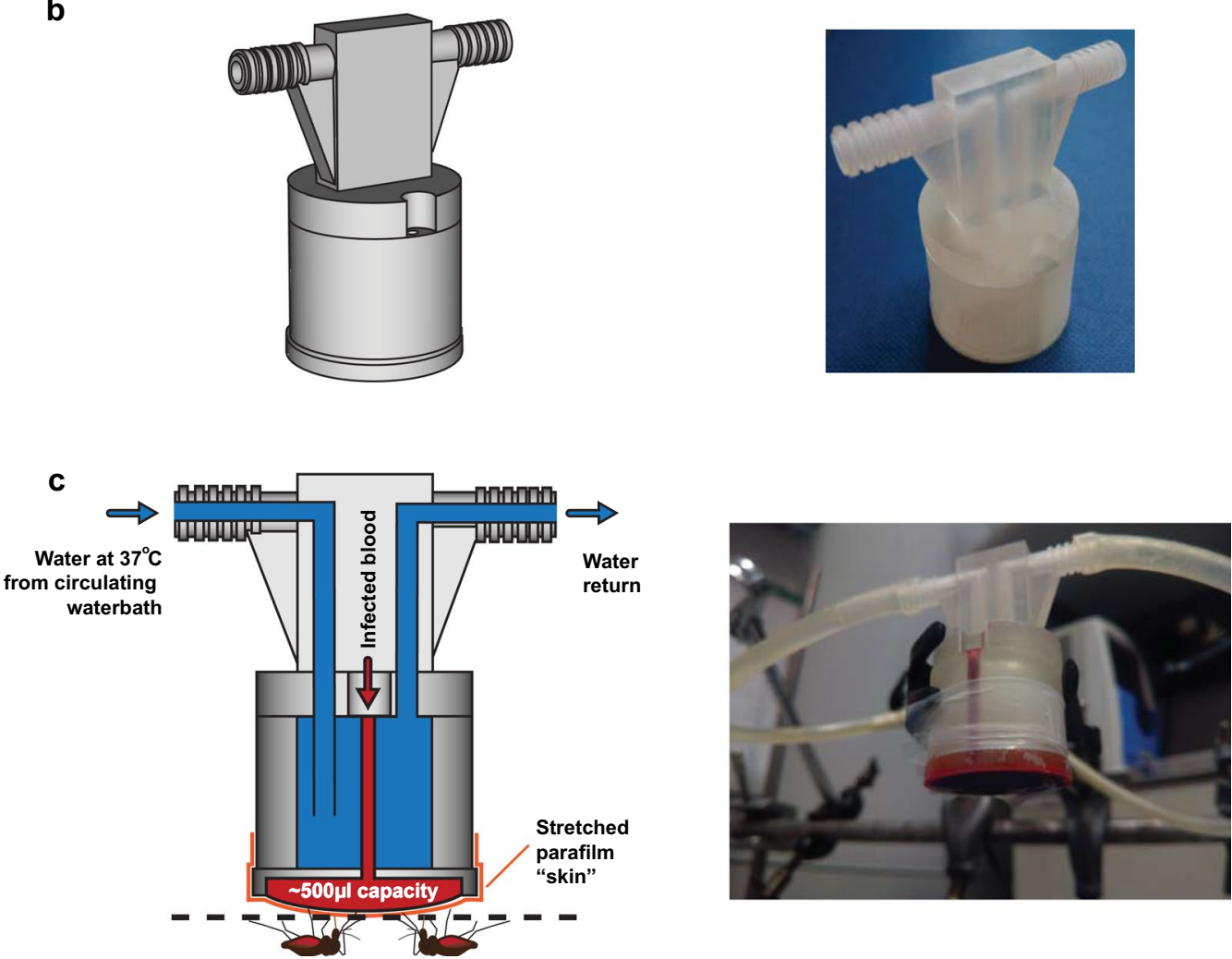

Fig. 1 Assembly and operation of the 3D-printed membrane feeder. a The membrane feeder was designed in two parts, a top chamber that connects to a circulating water bath and a bottom chamber holding a water reservoir and the RBC/gametocyte/serum sample on the underside. b Both pieces are glued together into a single, watertight unit. c When in operation, circulating warm water maintains the temperature of the gametocyte-infected blood sample that is injected through access holes and sits between a layer of stretched Parafilm ${ }^{\circledR}$ and the underside of the feeder

the surface of the feeder or printing at higher resolution. Furthermore, the 3D-printed feeder was fully functional even after a consecutive 7 day exposure to
$10 \%$ bleach solution - frequently used to decontaminate feeders in field-based experiments where unscreened blood is used. A final operational consideration 

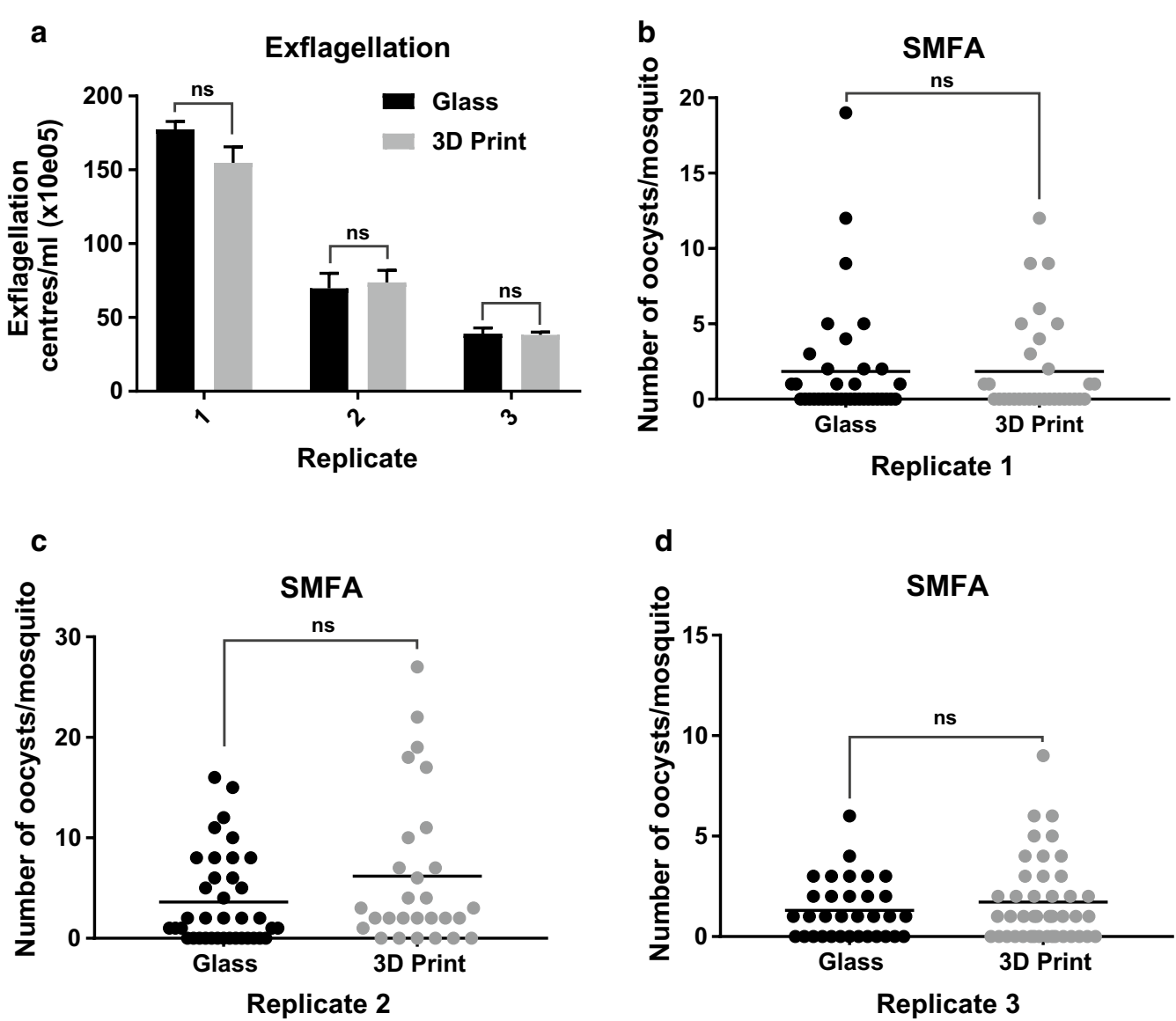

\begin{tabular}{|c|c|c|c|c|c|c|}
\cline { 2 - 7 } & \multicolumn{2}{|c|}{ Replicate 1 } & \multicolumn{2}{c|}{ Replicate 2 } & \multicolumn{2}{c|}{ Replicate 3 } \\
\cline { 2 - 7 } & glass & 3D print & glass & 3D print & glass & 3D print \\
\hline $\begin{array}{c}\text { Infection prevalence } \\
\text { (\% infected) }\end{array}$ & $41 \%$ & $41 \%$ & $63 \%$ & $79 \%$ & $61 \%$ & $63 \%$ \\
\hline $\begin{array}{c}\text { Oocyst intensity } \\
\text { (mean } \\
\text { oocyst/mosquito) }\end{array}$ & 1.8 & 1.8 & 3.6 & 6.2 & 1.3 & 1.7 \\
\hline \# midguts dissected & 37 & 32 & 38 & 28 & 33 & 43 \\
\hline
\end{tabular}

Fig. 2 Comparative P. falciparum SMFAs with a commercial glass feeder and 3D-printed feeder. a After feeding, blood was sampled from the feeders and exflagellation was induced and quantified. Exflagellation is indicated per millilitre per feeder. Three independent biological replicates are shown (1-3). No statistically significant difference was found. $\mathbf{b}-\mathbf{d}$ Three standard membrane feeding assays (SMFAs) were performed in which the RBC/gametocyte/serum sample was split between the two feeders, mosquitoes allowed to feed and midgut oocysts quantified 9 days later. Each dot represents one mosquito midgut. The mean oocyst number is indicated with a straight horizontal line. No statistically significant difference was found between the two feeders. e Infection parameters quantified from SMFA replicates 1-3

required to be optimised by the user is water bath temperature that may need to be decreased in tropical areas with high ambient temperatures to prevent heat damage to the gametocytes.

\section{Conclusions}

The 3D-printed feeder design enables researchers to inexpensively produce their own SMFA feeders as an alternative to expensive and fragile glass feeders that 

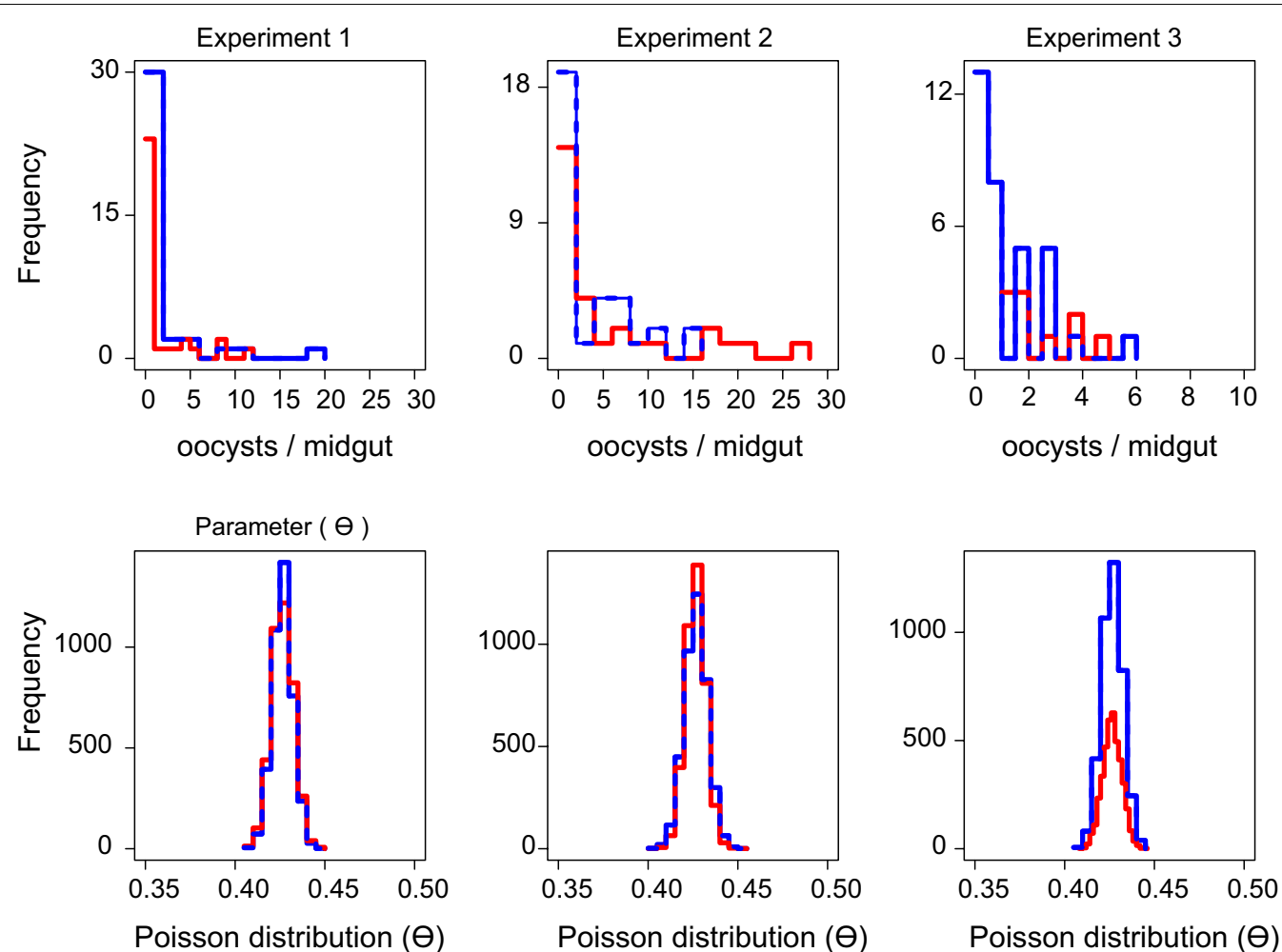

Poisson distribution $(\Theta)$

Poisson distribution $(\Theta)$
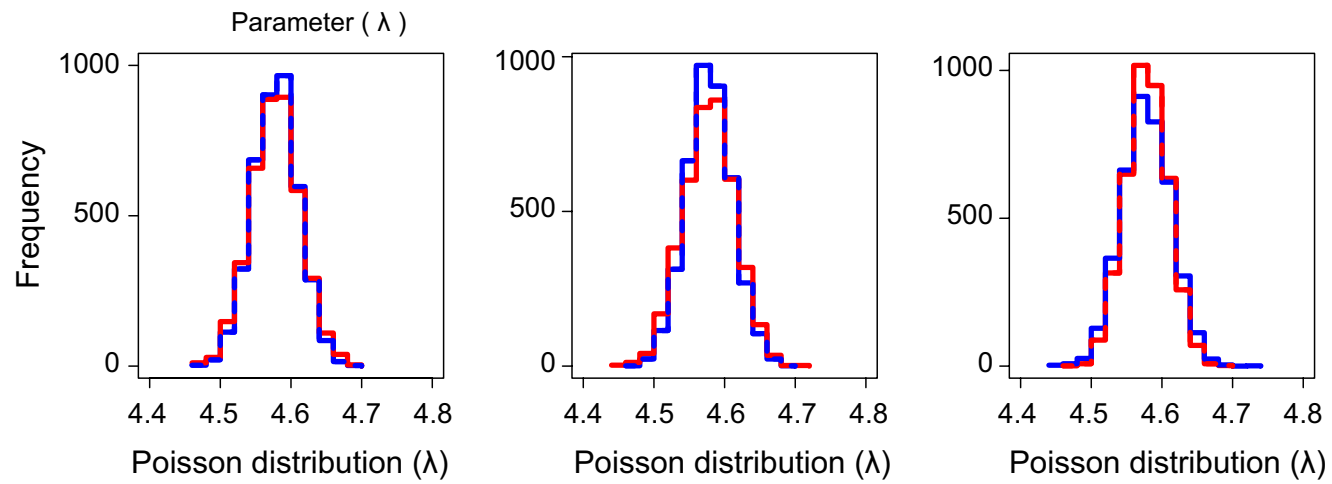

Glass feeder

- 3D printed feeder

Fig. 3 Statistical analysis of SMFA data. a Raw data for the number of oocysts per fed mosquito. These oocyst intensities were compared using a zero-inflated Poisson regression to accommodate the distribution of the count data. A Bayesian approach was used to demonstrate there is no statistical difference between experimental replicates. Zero-inflated Poisson probability distributions were fitted using Hamiltonian Monte Carlo sampling methods described in the main manuscript. There is a probability $\theta$ of drawing a zero and a probability $(1-\theta)$ of drawing from a Poisson distribution with mean parameter $\lambda$. Four chains were initialised to assess the convergence of 2000 iterations, the first 1000 were discarded as burn in. The posterior distributions of parameters (4000 iterations) $\mathbf{b} \theta$ and $\mathbf{c} \lambda$ for each experimental replicate (columns 1-3 respectively) are shown, posterior checks demonstrate the oocyst counts are not statistically different when mosquitoes feed on a glass (blue) or a 3D-printed (red) standard membrane feeding assay (SMFA) 
require specialist manufacturing. This new 3D-printed feeder can be used in a wide range of applications in addition to standard SMFAs, as it is not limited to the species used here. Application might include the assessment of vector competence for malaria [12], the epidemiological assessment of the infectious reservoir for malaria [13], clinical drug trials [14], and transmission-blocking studies $[15,16]$.

\section{Additional files}

Additional file 1. Feeder bottom. 3D CAD file of the bottom part of the membrane feeder.

Additional file $\mathbf{2}$ Feeder top. 3D CAD file of the top part of the membrane feeder.

\section{Abbreviations}

RBC: red blood cell; SMFA: standard membrane feeding assay.

\section{Authors' contributions}

MD designed the 3D membrane feeder; US provided the $P$. falciparum gametocytes; MT reared the Anopheles stephensi mosquitoes; KW performed the SMFAs; KW and MD analysed the data; ESS performed the statistical power calculations, JB, MD and KW wrote the manuscript. All authors read and approved the final manuscript.

\section{Author details}

${ }^{1}$ Department of Life Sciences, Imperial College, London SW7 2AZ, UK. ${ }^{2}$ MRC Centre for Global Infectious Disease Analysing, Department of Infectious Disease Epidemiology, Imperial College, London W2 1PG, UK.

\section{Acknowledgements}

We would like to thank our funders for supporting this work.

\section{Competing interests}

All authors declare that they have no competing interests.

\section{Consent for publication}

All authors consent to publication.

\section{Data availability}

The 3D modelling files are freely available for download and modification (Additional files 1, 2) under a Creative Commons 4.0 Attribution International license.

\section{Funding}

Research undertaken was supported through a Wellcome Investigator Award to JB (100993/Z/13/Z) and funding from the Medicines for Malaria Venture (MMV08/2800, MJD and JB).

\section{Publisher's Note}

Springer Nature remains neutral with regard to jurisdictional claims in published maps and institutional affiliations.

Received: 15 June 2018 Accepted: 30 July 2018

Published online: 03 August 2018

\section{References}

1. Billker O, Lindo V, Panico M, Etienne AE, Paxton T, Dell A, et al. Identification of xanthurenic acid as the putative inducer of malaria development in the mosquito. Nature. 1998;392:289-92.

2. Angrisano F, Sala KA, Da DF, Liu Y, Pei J, Grishin NV, et al. Targeting the conserved fusion loop of HAP2 inhibits the transmission of Plasmodium berghei and falciparum. Cell Rep. 2017;21:2868-78.

3. Miura K, Takashima E, Deng B, Tullo G, Diouf A, Moretz SE, et al. Functional comparison of Plasmodium falciparum transmission-blocking vaccine candidates by the standard membrane-feeding assay. Infect Immun. 2013;81:4377-82.

4. Delves M, Plouffe D, Scheurer C, Meister S, Wittlin S, Winzeler EA, et al. The activities of current antimalarial drugs on the life cycle stages of Plasmodium: a comparative study with human and rodent parasites. PLoS Med. 2012;9:e1001169.

5. Ouédraogo AL, Guelbéogo WM, Cohuet A, Morlais I, King JG, Gonçalves $B P$, et al. A protocol for membrane feeding assays to determine the infectiousness of P. falciparum naturally infected individuals to Anopheles gambiae. Malaria World J. 2013:4:1-4.

6. Hemotek membrane feeding systems — for blood sucking insects. http:// hemotek.co.uk/. Accessed 13 June 2018.

7. Mosquito Feeder Glassware Malaria Research-Chemglass Life Sciences. https://chemglass.com/pages/Mosquito_Feeder_Glassware. Accessed 13 June 2018.

8. Ponnudurai T, Lensen AH, Van Gemert GJ, Bensink MP, Bolmer M, Meuwissen $\mathrm{JH}$. Infectivity of cultured Plasmodium falciparum gametocytes to mosquitoes. Parasitology. 1989:98(Pt 2):165-73.

9. Ramakrishnan C, Delves MJ, Lal K, Blagborough AM, Butcher G, Baker KW, et al. Laboratory maintenance of rodent malaria parasites. Methods $\mathrm{Mol}$ Biol Clifton NJ. 2013;923:51-72.

10. Zeileis A, Kleiber C, Jackman S. Regression models for count data in R. J Stat Softw. 2008;27:1-25.

11. Papaspiliopoulos $O$, Roberts $G O$, Sköld M. A general framework for the parametrization of hierarchical models. Stat Sci. 2007;22:59-73.

12. Riehle MM, Bukhari T, Gneme A, Guelbeogo WM, Coulibaly B, Fofana A, et al. The Anopheles gambiae 2La chromosome inversion is associated with susceptibility to Plasmodium falciparum in Africa. eLife. 2017:6:e25813.

13. Gonçalves BP, Kapulu MC, Sawa P, Guelbéogo WM, Tiono AB, Grignard $L$, et al. Examining the human infectious reservoir for Plasmodium falciparum malaria in areas of differing transmission intensity. Nat Commun. 2017:8:1133.

14. Dicko A, Roh ME, Diawara H, Mahamar A, Soumare HM, Lanke K, et al. Efficacy and safety of primaquine and methylene blue for prevention of Plasmodium falciparum transmission in Mali: a phase 2, single-blind, randomised controlled trial. Lancet Infect Dis. 2018;18:627-39.

15. Blagborough AM, Sinden RE. Plasmodium berghei HAP2 induces strong malaria transmission-blocking immunity in vivo and in vitro. Vaccine. 2009:27:5187-94.

16. Armistead JS, Morlais I, Mathias DK, Jardim JG, Joy J, Fridman A, et al. Antibodies to a single, conserved epitope in Anopheles APN1 inhibit universal transmission of Plasmodium falciparum and Plasmodium vivax malaria. Infect Immun. 2014;82:818-29.

Ready to submit your research? Choose BMC and benefit from

- fast, convenient online submission

- thorough peer review by experienced researchers in your field

- rapid publication on acceptance

- support for research data, including large and complex data types

- gold Open Access which fosters wider collaboration and increased citations

- maximum visibility for your research: over 100M website views per year

At BMC, research is always in progress.

Learn more biomedcentral.com/submissions 